\title{
Estado nutricional, consumo alimentar e risco cardiovascular: um estudo em universitários ${ }^{1}$
}

\author{
Nutritional status, food consumption \\ and cardiovascular risk: a study \\ on university students
}

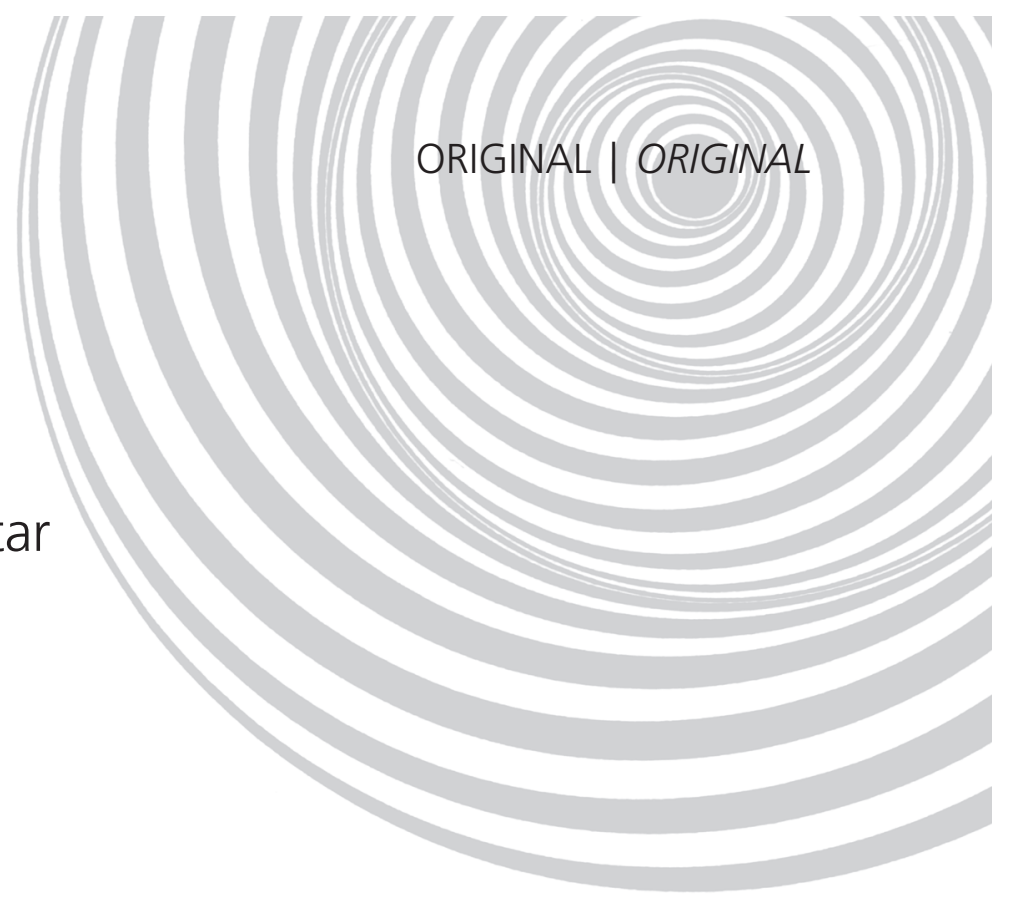

Marina de Moraes Vasconcelos PETRIBÚ2

Poliana Coelho CABRAL ${ }^{2}$

IIma Kruze Grande de ARRUDA ${ }^{2}$

\section{RE S U M O}

\section{Objetivo}

Descrever a proporção de fatores de risco para doenças cardiovasculares, dando ênfase aos fatores nutricionais, em alunos da área de saúde de uma universidade pública do Recife.

\section{Métodos}

Foram avaliados 250 estudantes por um questionário que abordou aspectos biossociais, dados sobre estilo de vida, história familiar para doenças cardiovasculares, variáveis antropométricas e consumo alimentar, avaliado pelo Recordatório de 24horas.

\section{Resultados}

Foi encontrada a seguinte freqüência para os fatores de risco analisados: tabagismo $(2,8 \%)$, sedentarismo $(41,7 \%)$, excesso de peso $(35,5 \%$ e $5,3 \%$ nos sexos masculino e feminino, respectivamente $p<0,01)$, história familiar de hipertensão (35,5\%), diabetes (11,3\%) e obesidade $(20,2 \%)$, morte antes dos 50 anos por doenças cardiovasculares nos familiares diretos $(14,8 \%)$. Com relação ao consumo alimentar, observou-se um elevado percentual de inadequação no consumo de energia e um baixo percentual de inadequação no consumo de proteínas e carboidratos. Quanto ao perfil lipídico da dieta, mais de 40,0\% dos estudantes apresentaram consumo de colesterol acima do recomendado e, em 17,9\% dos homens e 44,8\% das mulheres foi evidenciado um elevado consumo de gordura saturada $(p<0,01)$. O consumo de ácido linoléico, ácido graxo monoinsaturado e poliinsaturado mostrou-se insuficiente em mais de 95,0\% dos indivíduos estudados.

\footnotetext{
1 Apoio: Conselho Nacional de Desenvolvimento Científico e Tecnológico (processo no 478789/2007-6).

2 Universidade Federal de Pernambuco, Centro de Ciências da Saúde, Programa de Pós-Graduação em Nutrição. Av. Prof. Moraes Rego, 1235, Cidade Universitária, 50670-901, Recife, PE, Brasil. Correspondência para/Correspondence to: M.M.V. PETRIBÚ. E-mail: <mpetribu@hotmail.com>.
} 
838 | M.M.V. PETRIBÚ et al.

\section{Conclusão}

A alta proporção de fatores de risco cardiovasculares representa uma advertência, dada a juventude da população considerada, e mostra a necessidade de insistir em medidas educativas e de promoção de condutas preventivas.

Termos de indexação: Consumo alimentar. Doenças cardiovasculares. Estudantes de ciências da saúde.

\section{A B S T R A C T}

\section{Objective}

This study aimed to describe the proportion of risk factors for cardiovascular diseases, emphasizing nutritional factors, among health students from a public university in Recife, Brazil.

\section{Methods}

Two hundred and fifty students were assessed through a questionnaire that addressed biosocial aspects, lifestyle data, family history for cardiovascular diseases, anthropometric variables and food consumption determined by the 24-hour recall.

\section{Results}

The following rates were found for the assessed risk factors: smoking (2.8\%), inactivity (41.7\%), overweight (35.5\% among men and 5.3\% among women, $p<0,01$ ), family history of hypertension (35.5\%), diabetes (11.3\%), obesity (20.2\%) and death of close relatives before age 50 due to cardiovascular diseases (14.8\%). Regarding food consumption, a high percentage of individuals had inappropriate energy intake and a low percentage had inappropriate protein and carbohydrate intakes. Regarding the fat profile of the diet, more than $40.0 \%$ of the students consumed more cholesterol than the recommended levels and $17.9 \%$ of the men and $44.8 \%$ of the women consumed high amounts of saturated fat $(p<0.01)$. The consumption of linoleic acid and monounsaturated and polyunsaturated fatty acids was inadequate in more than 95\% of the individuals under study.

\section{Conclusion}

The high rates of risk factors are a warning sign, given the young age of the studied population, and show the need to insist on measures to prevent primary cardiovascular disease.

Indexing terms: Food consumption. Cardiovascular diseases. Students, health occupations.

\section{N T R O D U Ç Ã O}

As doenças cardiovasculares representam a principal causa de mortalidade no Brasil desde os anos 60, ou seja, em números de hoje, cerca de 300 mil brasileiros por ano são vítimas desse mal ${ }^{1}$. Quanto à sua etiologia, os principais fatores de risco foram identificados por estudos epidemiológicos realizados nos últimos 30 anos, destacando-se o tabagismo, a hipertensão arterial e a hipercolesterolemia seguidos por diabetes, obesidade e inatividade física ${ }^{2,3}$.

Segundo Oliveira \& Escrivão ${ }^{4}$, os fatores genéticos parecem ser fundamentais na determinação das condições clínicas citadas acima, mas as variações fenotípicas são estabelecidas a partir da interação com o meio ambiente, no qual o hábito alimentar tem papel de destaque. De fato, pesquisadores do mundo todo têm se voltado cada vez mais para a possível relação da nutrição com as doenças cardiovasculares, e os estudos têm demonstrado a estreita relação entre a causalidade das Doenças Crônicas não Transmissíveis (DCNT) e os fatores da alimentação ${ }^{5,6}$.

Várias evidências demonstram que o processo aterosclerótico se inicia na infância e leva décadas para avançar ${ }^{7,8}$, demonstrando que as raízes das doenças cardiovasculares em adultos e idosos têm sua base na infância. Nobre et al. ${ }^{8}$, em 2006, encontraram uma prevalência de 24,0\% de excesso de peso, 53,3\% de hábito alimentar inadequado, 15,4\% de sedentarismo, 62,6\% de consumo de álcool e 23,1\% de tabagismo, em 
alunos do ensino fundamental. Avaliando estudantes universitários, Vieira et al..$^{9}$ relataram que $73,5 \%$ dos indivíduos consumiam bebida alcoólica, 57,0\% não realizavam atividade física, 7,0\% fumavam e $58,7 \%$ apresentavam percentual de gordura corporal elevado.

Para muitos estudantes, o ingresso na faculdade corresponde ao primeiro momento em que eles terão de se responsabilizar por sua moradia, alimentação e gestão de suas finanças. $A$ inabilidade para realizar tais tarefas, juntamente com fatores psicossociais, estilo de vida e situações próprias do meio acadêmico, podem resultar em omissão de refeições, consumo de lanches rápidos e ingestão de refeições nutricionalmente desequilibradas ${ }^{9,10}$.

Em todas as etapas da vida humana cabem diversas ações de promoção da saúde, voltadas para os indivíduos, para os grupos sociais e para as populações. No entanto, pelo exposto acima, verifica-se que essas ações merecem maior atenção e cuidado em indivíduos jovens, visando à prevenção primária das doenças cardiovasculares, ou seja, à ação antes que o indivíduo adoeça. Desse modo, a finalidade principal deste trabalho é conhecer a freqüência de alguns fatores de risco ambientais, dando a ênfase aos fatores nutricionais, em uma população de adultos jovens constituída por alunos da área de saúde de uma universidade pública do Recife, com o objetivo de planejar estratégias de prevenção e de combate a esse mal.

\section{M É T O D O S}

Estudo do tipo transversal, com base no modelo epidemiológico descritivo, em estudantes regularmente matriculados nos cursos da área de saúde de uma universidade pública da cidade do Recife, onde foram avaliados o consumo energético e dos macronutrientes e alguns fatores de risco para o desenvolvimento de doenças cardiovasculares. O tamanho amostral foi determinado por um estudo piloto, no qual foi estimada a prevalência do excesso de peso, o qual dentre todos os fatores de risco foi o que se mostrou como tendo menor prevalência nesta população. Desse modo, adotando-se uma prevalência de 17\% (encontrada no estudo piloto), com uma margem de erro aceitável de 5\%, uma confiabilidade de 95\% e uma população em torno de 4000 alunos, o tamanho amostral ficou em torno de 206 estudantes. Para corrigir eventuais perdas, esse valor foi corrigido em $20 \%$, totalizando uma amostra em torno de 247 indivíduos. A necessidade da realização do projeto piloto vem do fato de que a prevalência do excesso de peso, relatada na literatura, foi obtida de populações do Sul e Sudeste do Brasil, com hábitos alimentares, provavelmente, bem diferentes dos observados na na Região Nordeste.

As variáveis biossociais verificadas foram: sexo e idade dos estudantes e escolaridade dos pais. Foram coletados também dados sobre estilo de vida e história familiar para doenças cardiovasculares, tais como: prática de atividade física, hábito de fumar, ocorrência de hipertensão arterial, diabetes mellitus e/ou obesidade nos pais, além da ocorrência de morte por doença cardiovascular em um familiar direto (pais, tios ou avós), com idade inferior a 50 anos. Para a categorização da atividade física, os estudantes preencheram um questionário com o número aproximado de horas diárias que passam desempenhando atividades de repouso (dormir ou descansar), muito leves (sentados ou em atividades paradas), leves (como andar em superfície reta de 4 a $5 \mathrm{~km} / \mathrm{h}$ ), moderadas (como andar de 6 a $7 \mathrm{~km} / \mathrm{h}$ ) e intensas (como andar com peso em superfície inclinada) $)^{11}$, sendo essas horas multiplicadas por 1,0, 1,5, 2,5, 5,0 e 7,0, respectivamente, e somadas ao final. O valor final foi então dividido por 24 e os indivíduos que obtiveram um nível de atividade física entre $\geq 1,0$ e $<1,4$ foram classificados como sedentários, $\geq 1,4$ e $<1,6$ como pouco ativo, $\geq 1,6$ e $<1,9$ ativo e $\geq 1,9$ e $<2.5$ muito ativo, de acordo com os critérios do Institute of Medicine ${ }^{12}$.

Para avaliar o estado nutricional foi utilizado o Índice de Massa Corporal (IMC) sendo que 
a classificação utilizada foi a proposta pela Organização Mundial de Saúde em $1995^{13}$. Quanto às variáveis antropométricas (peso e altura), os dados utilizados foram os referidos pelos estudantes, o que têm sido utilizado em estudos epidemiológicos, principalmente por favorecer a economia de recursos e a simplificação do trabalho de cam$\mathrm{po}^{14}$. De acordo com os resultados de alguns estudos, o peso e a altura referidos apresentam níveis aceitáveis de validade, até mesmo entre os obesos (que poderiam apresentar maior tendência à subestimação do peso) e entre grupos com baixa escolaridade ${ }^{14,15}$.

A avaliação do consumo energético e de macronutrientes foi realizada por recordatório de 24 horas. Como o registro dos alimentos foi feito em medidas caseiras, houve a necessidade de conversão destas em gramas, utilizando-se como padrão de referência, a Tabela de Pinheiro et al. ${ }^{16}$.

Além da análise em termos de valor energético, proteínas, carboidratos e gorduras, foi analisado também o perfil lipídico da dieta (colesterol, gordura saturada, ácidos graxos poliinsaturados, monoinsaturados e ácido linoléico). Para estimar a percentagem de inadequação das dietas em relação ao consumo enegético, protéico e glicídico foram considerados os valores da Dietary Reference Intakes (DRI) propostos pelo Food and Nutrition Board (FND) ${ }^{12}$. Com o objetivo de determinar a variação intrapessoal do consumo alimentar foram aplicados mais dois Recordatórios de $24 \mathrm{~h}$ em $20 \%$ dos estudantes avaliados, sorteados aleatoriamente. Estes novos inquéritos foram realizados com intervalo, de pelo menos, 15 dias entre as coletas, repetindo o procedimento adotado no primeiro dia de Recordatório de 24 horas. O ajuste da distribuição da ingestão de energia, proteínas e carboidratos foi realizado com a remoção do efeito da variabilidade intra-individual, pelo método proposto pelo lowa State University ${ }^{17,18}$. A proporção de inadequação da ingestão de energia, proteínas e carboidratos correspondeu à proporção de indivíduos cujo consumo estava abaixo da Necessidade de Energia Estimada (Estimated Energy Requirement) e da Necessidade
Média Estimada (Estimated Average Requirement) estabelecida para energia e proteínas e carboidratos, respectivamente ${ }^{12}$.

Como o lipídio e suas frações ainda não possuem uma necessidade média estimada, só ingestão adequada, não foi possível fazer a estimativa da proporção de inadequação. Neste caso, a avaliação foi realizada tomando-se como referência os intervalos de distribuição aceitável dos nutrientes, estabelecidos em função da prevenção das doenças crônicas não transmissíveis e das necessidades nutricionais ${ }^{12}$.

A construção do banco de dados e a análise estatística foram realizadas nos programas Epi Info versão 6,04 e SPSS. As variáveis contínuas foram testadas quanto à normalidade da distribuição, pelo teste de Kolmogorov Smirnov, e aquelas que não obtiveram distribuição normal (energia e lipídios no sexo feminino) foram convertidas para o seu logaritmo natural e testadas novamente, sendo verificada a normalidade. Os dados foram descritos em média, desvio-padrão e percentuais de ingestão.

Para verificar associações entre as variáveis dicotômicas, foi aplicado o teste do qui-quadrado com correção de Yates. Na descrição das proporções, a distribuição binomial foi aproximada à distribuição normal pelo Intervalo de Confiança de $95 \%$. A comparação entre as médias foi realizada pelo teste $t$ de Student (duas médias). Foi adotado o nível de significância de 5\% para rejeição da hipótese de nulidade.

A análise da composição da dieta foi realizada utilizando o software de apoio à Nutrição da Escola Paulista de Medicina ${ }^{19}$. A tabela base deste programa é a do Departamento de Agricultura dos Estados Unidos, ano 1976-1986. Desse modo, em virtude da ocorrência de inúmeros produtos de consumo regional, alguns alimentos tiveram que ser acrescidos, sendo utilizada a Tabela Brasileira de Composição de Alimentos $(\text { TACO })^{20}$.

O estudo foi pautado pelas normas éticas para pesquisa envolvendo seres humanos, cons- 
tantes da resolução 196 do Conselho Nacional de Saúde, e foi submetido e aprovado pelo Comitê de Ética da Universidade Federal de Pernambuco (Registro CEP/CCS/UFPE No 101/06). Os alunos que concordaram em participar receberam todas as informações sobre o estudo e assinaram o Termo de Consentimento Livre e Esclarecido.

\section{RESULTADOS}

A amostra $(n=250)$ foi constituída por $61,6 \%$ de mulheres com média de idade próxima dos 22 anos. Neste estudo, a renda familiar não foi coletada, utilizando-se como proxi o grau de instrução do pai e da mãe na categorização do perfil socioeconômico dos estudantes (Tabela 1).

Na Tabela 1 encontram-se descritas as características biossociais, do estilo de vida e da história familiar dos estudantes avaliados. Foi observada uma altura média de 1,77m (Desvio-Padrão - $D P=0,1)$ e 1,62m ( $D P=0,6)$, em homens e mulheres, respectivamente.

Com relação à atividade física, foi encontrada uma diferença estatisticamente significante entre o percentual de indivíduos sedentários e pouco ativos entre os sexos, sendo esta proporção bem superior no sexo feminino $(p<0,05)$. A média de IMC observada foi de $20,9 \mathrm{~kg} / \mathrm{m}^{2}$ e $23,9 \mathrm{~kg} / \mathrm{m}^{2}$ para o sexo feminino e masculino, respectivamente, sendo a diferença estatisticamente significante $(p<0,01)$ (Tabela 2 ). A proporção de excesso de peso encontrada nas mulheres foi significantemente menor do que nos homens $(5,3 \% \times 35,5 \%, p<0,01)$.

A ingestão diária e o percentual de inadequação de energia, proteínas e carboidratos

Tabela 1. Características biossociais, do estilo de vida e da história familiar para doença cardiovascular de estudantes universitários. Recife (PE), 2007

\begin{tabular}{|c|c|c|c|}
\hline Variáveis & $n=250$ & $\%$ & IC $95 \%{ }^{*}$ \\
\hline \multicolumn{4}{|l|}{ Sexo } \\
\hline Masculino & 96 & 38,4 & $32,4-44,8$ \\
\hline Feminino & 154 & 61,6 & $55,2-67,6$ \\
\hline \multicolumn{4}{|l|}{ Tabagismo } \\
\hline Sim & 7 & 2,8 & $1,2-5,9$ \\
\hline Não & 243 & 97,2 & $94,1-98,8$ \\
\hline \multicolumn{4}{|l|}{ Atividade fisica } \\
\hline Sedentário & 104 & 41,7 & $35,5-48,0$ \\
\hline Pouco ativo & 86 & 34,4 & $28,6-40,7$ \\
\hline Moderadamente ativo & 51 & 20,2 & $15,7-26,0$ \\
\hline Muito ativo & 9 & 3,6 & $1,8-6,9$ \\
\hline \multicolumn{4}{|l|}{ Instrução dos pais } \\
\hline Até $1^{\circ}$ grau & 32 & 12,7 & $9,0-17,7$ \\
\hline Até $2^{\circ} \mathrm{grau}$ & 93 & 37,3 & $31,2-43,5$ \\
\hline Superior & 125 & 50,0 & $43,6-56,3$ \\
\hline \multicolumn{4}{|l|}{ Antecedentes familiares } \\
\hline Hipertensão (pai e/ou mãe) & 88 & 35,2 & $29,4-41,5$ \\
\hline Diabetes (pai e/ou mãe) & 28 & 11,2 & $7,7-15,9$ \\
\hline Obesidade (pai e/ou mãe) & 50 & 20,0 & $15,3-25,6$ \\
\hline Nenhuma doença (pai e/ou mãe) & 84 & 33,6 & $27,8-39,9$ \\
\hline \multicolumn{4}{|c|}{ Parente com morte antes dos 50 anos por doença cardiovascular } \\
\hline Sim & 37 & 14,8 & $10,8-19,9$ \\
\hline Não & 213 & 85,2 & $80,0-89,2$ \\
\hline
\end{tabular}

${ }^{*} \mathrm{IC}=$ intervalo de confiança. 
encontram-se descritos na Tabela 3. Observou-se uma alta freqüência de inadequação de energia, não havendo diferença estatística entre os sexos ( $p>0,05)$. Apesar disso, neste estudo, o percentual de indivíduos que tinham um consumo superior à necessidade energética estimada, mais 2 desvios-padrão, ficou em torno de 15,8\% e 14,3\% para mulheres e homens, respectivamente. Ao contrário da energia, foi observado um baixo percentual de inadequação de proteínas e carboidratos.

Do ponto de vista dos nutrientes relacionados às doenças cardiovasculares, analisados a partir de dados do consumo alimentar, chamam especial atenção o alto consumo de colesterol (>300mg/dia), Figura 1, por 40,4\% dos homens e $40,9 \%$ das mulheres ( $p>0,05)$ e o consumo de gordura saturada superior à recomendação (>10\% do valor energético total) por 17,9\% dos homens e $44,8 \%$ das mulheres, sendo este último diferencial estatisticamente significante $(p<0,01)$.

O consumo de ácido graxo linoléico $(5,6$, $\mathrm{DP}=7,8 \mathrm{~g}$ e 4,2, $\mathrm{DP}=3,9 \mathrm{~g}$ para homens e mulheres, respectivamente) foi bastante inferior à recomendação de 17 g/dia para homens e 12g/dia para

Tabela 2. Classificação antropométrica de universitários, segundo o índice de massa corporal. Recife (PE), 2007.

\begin{tabular}{|c|c|c|c|c|c|}
\hline \multirow[t]{2}{*}{ Variável (pontos de corte) } & \multicolumn{2}{|c|}{$\begin{array}{c}\text { Homens } \\
(n=96)\end{array}$} & \multicolumn{2}{|c|}{$\begin{array}{c}\text { Mulheres } \\
(n=154)\end{array}$} & \multirow[t]{2}{*}{$p$} \\
\hline & $\mathrm{n}$ & $\%$ & $n$ & $\%$ & \\
\hline \multicolumn{6}{|l|}{$I M C\left(\mathrm{~kg} / \mathrm{m}^{2}\right)$} \\
\hline Baixo peso $(<18,5)$ & 2 & 2,2 & 18 & 11,8 & $<0,05$ \\
\hline Eutrófico $(18,5$ a 24,9$)$ & 60 & 62,4 & 128 & 82,9 & $<0,01$ \\
\hline Excesso de peso $(\geq 25,0)$ & 34 & 35,5 & 8 & 5,3 & $<0,01$ \\
\hline
\end{tabular}

IMC: índice de massa corporal. mulheres ${ }^{12}$. Praticamente todos os indivíduos estudados (95,7\% dos homens e 95,4\% das mulheres) apresentaram um consumo de ácido graxo linoléico abaixo do recomendado, o que também foi observado com relação aos ácidos graxos mono e poliinsaturados (Figura 2).

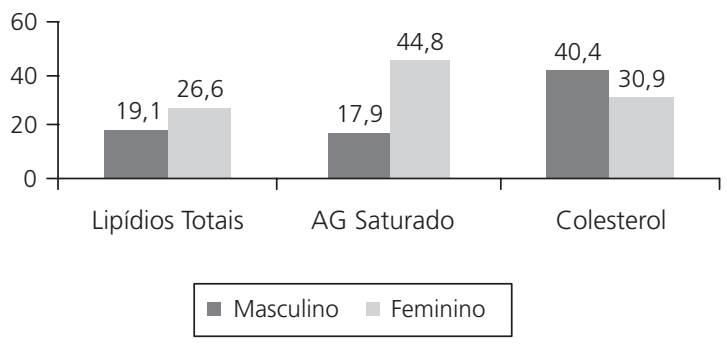

Figura 1. Distribuição percentual de universitários da cidade do Recife, segundo o sexo, quanto ao consumo acima do recomendado de nutrientes de risco para doenças cardiovasculares. Recife (PE), 2007.

Nota: \% de consumo acima do recomendado homens $x$ mulheres lipídios totais $\chi^{2}=1,62(p>0,05)$; ácido graxo saturado $\chi^{2}=$ $18,01(p<0,01)$; colesterol $\chi^{2}=1,93(p>0,05)$. AG: ácido graxo.



Figura 2. Distribuição percentual de universitários da cidade do Recife, segundo o sexo, quanto ao consumo abaixo do recomendado de nutrientes protetores para doenças cardiovasculares. Recife (PE), 2007.

Nota: AG: ácido graxo

Tabela 3. Ingestão diária e percentual de inadequação (\%Inad) de energia e macronutrientes de universitários. Recife (PE), 2007.

\begin{tabular}{|c|c|c|c|c|c|c|c|c|c|c|}
\hline \multirow[t]{2}{*}{ Nutrientes } & \multicolumn{2}{|c|}{$\begin{array}{l}\text { Recomendação*12 } \\
\text { Sexo Masculino }\end{array}$} & \multicolumn{3}{|c|}{$\begin{array}{c}\text { Sexo } \\
\text { Masculino }\end{array}$} & \multicolumn{2}{|c|}{$\begin{array}{l}\text { Recomendação*12 } \\
\text { Sexo Feminino }\end{array}$} & \multicolumn{3}{|c|}{$\begin{array}{c}\text { Sexo } \\
\text { Feminino }\end{array}$} \\
\hline & M & DP & M & DP & $\%$ & M & $\mathrm{DP}$ & $M$ & DP & $\%$ \\
\hline Energia (kcal) & 2889 & 354 & 2757 & 530 & 40,1 & 2056 & 235 & 1818 & 400 & 27,8 \\
\hline Proteína (g) & \multicolumn{2}{|c|}{49,4} & 118,5 & 35,1 & 2,4 & \multicolumn{2}{|c|}{36,4} & 78,0 & 14,9 & 0,3 \\
\hline Carboidrato (g) & \multicolumn{2}{|c|}{100} & 350,0 & 124,1 & 2,2 & \multicolumn{2}{|c|}{100} & 254,4 & 62,1 & 0,7 \\
\hline
\end{tabular}

*Institute of Medicine ${ }^{12}$.

M: Média; DP: desvio-padrão; \% percentual de inadequação. Análise: \% de inadequação homens x mulheres energia $\chi^{2}=3,16(p=0,0755)$. 


\section{DIS CUSS Ã O}

A predominância do sexo feminino observada não é nenhuma surpresa, tendo em vista que esse é um fato comum em vários cursos da área de saúde, como Nutrição, Enfermagem, Fonoaudiologia, entre outros.

Os resultados encontrados (Tabela 1) mostram que o fato de conseguir ingressar em uma universidade já se constitui em um processo de seleção, tendo em vista que, aproximadamente, $50 \%$ dos pais desses alunos apresentavam nível superior, sugerindo uma situação favorável em termos de acesso a macro e micronutrientes. De fato, a altura média dos estudantes que participaram deste estudo foi similar à observada por Fausto et al. ${ }^{21}$, estudando o perfil dos usuários de um restaurante universitário da Universidade Estadual Paulista (1,78, DP=0,08 e 1,63, DP=0,07 em homens e mulheres, respectivamente) e bem superior à média observada pela Pesquisa Nacional sobre Alimentação e Nutrição ${ }^{22}$ para o País como um todo, o que também é um fator que indiretamente reflete o bom nível socioeconômico desses estudantes.

No que se refere aos fatores de risco associados ao desenvolvimento de doenças cardiovasculares, observou-se no estudo uma baixa freqüência de tabagismo (2,8\%) (Tabela 1), quando comparada com outros estudos em universitários, que mostraram uma prevalência do hábito de fumar variando entre $7 \%$ a $27 \%$, 23 .

$\mathrm{O}$ alto percentual de estudantes sedentários e pouco ativos, superior no sexo feminino, foi similar aos resultados descritos por outros autores $^{23-25}$. Guedes ${ }^{25}$, analisando os níveis de prática de atividade física habitual em adolescentes, encontrou que os meninos foram consistentemente mais ativos fisicamente do que as meninas. Por volta de $54 \%$ dos meninos envolvidos no estudo de Guedes et al. ${ }^{25}$ foram classificados como ativos ou moderadamente ativos, enquanto aproximadamente $65 \%$ das meninas analisadas mostraram ser inativas ou muito inativas.

Um dos fatores que pode contribuir para o sedentarismo nos estudantes universitários da área de saúde é a falta de tempo, visto se tratar de cursos ministrados em horário integral (manhã e tarde). No entanto, esta situação se torna controversa ao pensar que, por serem estudantes da área de saúde, estes têm o total conhecimento da importância da prática regular de atividade física na qualidade de vida e na promoção da saúde, atuando inclusive como um fator protetor contra o aparecimento de doenças cardiovasculares.

É interessante observar que, apesar de praticar menos atividade física, sabendo ser esta um importante fator que auxilia na perda e no controle do peso corpóreo, as mulheres apresentaram um valor médio de IMC inferior ao encontrado para os homens, o que também se reflete em termos percentuais (Tabela 2). Esta menor freqüência de excesso de peso no sexo feminino também foi descrita por outros autores ${ }^{9,26}$.

Esta baixa proporção de excesso de peso no sexo feminino é um dado interessante visto que, segundo dados da Pesquisa de Orçamentos Familiares ${ }^{27}$, cerca de $40,0 \%$ dos indivíduos adultos do País apresentam excesso de peso. Talvez os valores encontrados neste estudo reflitam o status socioeconômico, pois a busca por um corpo magro é muito comum entre as mulheres pertencentes às classes sociais mais favorecidas $^{28}$. Além disso, distúrbios do comportamento alimentar associados ao temor de engordar, mais freqüentes entre adolescentes do sexo feminino e em mulheres mais jovens, poderiam estar contribuindo para o achado, visto que o percentual de baixo peso encontrado nas mulheres $(11,8 \%)$ é classificado como desnutrição moderada e considerado superior à fração esperada de indivíduos constitucionalmente magros (até 5,0\%) ) $^{27}$. Por outro lado, parte dos meninos pode estar sendo influenciada pelo modismo já disseminado em países desenvolvidos do culto ao corpo musculoso, o que pode mascarar sua verdadeira condição nutricional pelo IMC28.

Visto que os fatores genéticos representam cerca de $25 \%$ da etiologia das doenças cardiovasculares, e que estudos têm observado uma relação 
entre história familiar de risco e doença cardiovascular ${ }^{23}$, torna-se importante a análise da prevalência de morte antes dos 50 anos por doença cardiovascular e de doenças associadas às cardiovasculares em parentes próximos. No estudo, foi observada uma proporção de hipertensão, diabetes e obesidade de 35,5\%, 11,3\% e 20,2\%, respectivamente, para, pelo menos, um dos pais (Tabela 1), sendo inferior à encontrada por Girotto et al. ${ }^{23}$ na Argentina, que foi de $42,1 \%, 26,9 \%$ e $27,2 \%$, respectivamente. Foi observado que $14,8 \%$ dos estudantes relataram morte antes dos 50 anos por doença cardiovascular em algum parente próximo (Tabela 1). Dada a alta prevalência dos fatores de risco relacionados à história familiar, reforça-se ainda mais a importância do planejamento de ações que visem a diminuir a prevalência de fatores de risco exógenos, já que estes contribuem com cerca de 75,0\% com a etiologia das doenças cardiovasculares, e podem contribuir para a diminuição da prevalência de doença cardiovascular no futuro, por se tratar de indivíduos jovens.

Os estudos epidemiológicos têm fornecido evidências sobre a importância da dieta como fator de risco para doenças cardiovasculares. Vários alimentos e nutrientes têm sido relacionados à ocorrência de doenças crônicas em diferentes populações, destacando-se o consumo excessivo de colesterol e gorduras saturadas, nutrientes implicados na gênese das doenças cardiovasculares $^{29}$.

Um dos maiores problemas relacionados à estimativa do consumo alimentar é a subestimação da ingestão energética relatada pelos indivíduos, pois esta tem grandes implicações no tratamento e no controle da obesidade e de suas complicações metabólicas associadas ${ }^{30}$. Esta subestimação explica, em parte, o alto percentual de inadequação observado com relação ao consumo de energia. O baixo percentual de inadequação de proteínas observado pode ser justificado pelo aumento nas três últimas décadas, do consumo de carnes, leite e derivados entre os brasileiros ${ }^{27}$. O baixo percentual de inadequação de carboidratos também pode ser explicado pela tendência verificada nos resultados da Pesquisa de Orçamentos Familiares ${ }^{27}$, que mostra uma elevação no consumo de alguns alimentos ricos em carboidratos simples, como os biscoitos e refrigerantes. Por outro lado, foi observada uma tendência inversa no consumo de arroz (redução de $23 \%$ ), feijões e das demais leguminosas (redução de $30 \%$ ) e de raízes e tubérculos (redução de quase $50 \%$ ), alimentos ricos em carboidratos complexos $^{27}$.

O alto consumo de colesterol foi superior ao encontrado por Lopes et al. ${ }^{29}$, em seu estudo com adultos, de base populacional, que encontrou um consumo de colesterol superior a $300 \mathrm{mg} / \mathrm{dia}$ em $11,8 \%$ dos homens, enquanto que todas as mulheres tiveram ingestão adequada. A Pesquisa de Orçamentos Familiares ${ }^{27}$ encontrou um percentual médio de ácidos graxos saturados inferior ao limite máximo recomendado de $10,0 \%$ das calorias totais, sendo este teor superior no meio urbano (8,9\%). Este teor aumentava intensamente com o rendimento salarial, sendo o limite máximo virtualmente alcançado com os rendimentos mensais familiares per capita entre 2 e 5 salários-mínimos $(9,7 \%)$ e, claramente ultrapassados para famílias com rendimentos de mais de 5 salários mínimos per capita (11,2\% do total energético ${ }^{27}$. Lopes et al. ${ }^{29}$ observou um excesso no consumo de ácidos graxos saturados, sendo que $22,0 \%$ dos adultos estudados apresentaram uma ingestão de gorduras saturadas acima da recomendação máxima de $10,0 \%$ da energia total.

Os baixos consumos de ácido graxo linoléico, considerado um ácido graxo essencial, e dos ácidos graxos mono e poliinsaturados, que possuem importante ação hipocolesterolêmica, contribuem para a observação da baixa qualidade da alimentação desses indivíduos, o que, em longo prazo, pode contribuir para o aparecimento de doenças cardiovasculares.

\section{O N C L U S Ã O}

A alta freqüência de fatores de risco cardiovasculares representa uma advertência, dada 
a juventude da população considerada, e mostra a necessidade de insistir em medidas educativas e de promoção de condutas preventivas.

\section{COLABORADORES}

Todos os autores participaram ativamente de todas as etapas do trabalho, desenho do estudo, coleta dos dados, análise e interpretação dos resultados, redação do manuscrito e aprovação da versão final.

\section{REFERÊ N CIAS}

1. Costa RS, Silva CS. Doenças cardiovasculares. In: Cuppari L. Guia de medicina ambulatorial e hospitalar - UNIFESP - Escola Paulista de Medicina: nutrição clínica no adulto. 2a. ed. São Paulo: Manole; 2005. p.287-312.

2. Dawber TR. The Framingham study. Cambridge: Harvard University Press; 1980.

3. Moreira RO, Santos RD, Martinez L, Saldanha FC, Pimenta JLAC, Feijoo J, et al. Perfil lipídico de pacientes com alto risco para eventos cardiovasculares na prática clínica diária. Arq Bras Endocrinol Metabol. 2006; 50 (3):481-9. doi: 10.1590/5000427302006000300011.

4. Oliveira FLC, Escrivão MAMS. Prevenção das doenças do adulto na infância e na adolescência. In: Lopez FA, Brasil ALD. Nutrição e dietética em clínica pediátrica. São Paulo: Atheneu; 2004. p.105-24.

5. Steemburgo T, Dall'Alba V, Goss JL, Azevedo MJ. Fatores dietéticos e síndrome metabólica. Arq Bras Endocrinol Metabol. 2007; 51(9):1425-33. doi: 10.1590/S0004-27302007000900004.

6. Santos CRB, Portella ES, Ávila SS, Soares EA. Fatores dietéticos na prevenção e tratamento de comorbidades associadas à síndrome metabólica. Rev Nutr. 2006; 19(3):389-401. doi: 10.1590/\$14 15-52732006000300010.

7. Obarzanek E, Kimm SY, Barton BA, Van Horn LL, Kwiterovich PO Jr, Simons-Morton DG, et al. Longterm safety and efficacy of a cholesterol-lowering diet in children with elevated low-density lipoprotein cholesterol: seven-year results of the Dietary Intervention Study in Children (DISC). Pediatrics. 2001; 107(2):256-64. doi: 10.1542/ peds.107.2.256.

8. Nobre MRC, Domingues RZL, Silva AR, Colugnati FAB, Taddei JAAC. Prevalências de sobrepeso, obesidade e hábitos de vida associados ao risco cardiovascular em alunos do ensino fundamental. Rev Assoc Med Bras. 2006; 52 (2):118-24. doi: 10.1590/S0104-42302006000200023.

9. Vieira VCR, Priore SE, Ribeiro SMR, Franceschini SCC, Almeida LP. Perfil socioeconômico, nutricional e de saúde de adolescentes recém-ingressos em uma universidade pública brasileira. Rev Nutr. 2002; 15(3):273-82. doi: 10.1590/\$1415-527320 02000300003.

10. Cota RP, Miranda LS. Associação entre constipação intestinal e estilo de vida em estudantes universitários. Rev Bras Nutr Clin. 2006; 21(4):296-301.

11. National Research Council. Recommended Dietary Allowances.10th ed. Washington (DC): National Academy Press; 1989. p.27.

12. Institute of Medicine of the National Academies. Dietary references intakes for energy, carbohydrate, fiber, fat, fatty acids, cholesterol, protein, and amino acids. Washington (DC): National Academies Press; 2002.

13. World Health Organization. Physical status: the use and interpretation of anthropometry. Geneve: WHO; 1995. WHO Technical Report Series, n. 854.

14. Chor D, Coutinho ESF, Laurent R. Reliability of self reported weight and among state bank employees in Rio de Janeiro. Rev Saúde Pública. 1999; 33(1): 16-23. doi: 10.1590/S0034-891019990001000 04.

15. Fonseca MJM, Faerstein E, Chor D, Lopes SC. Validade de peso e estatura informados e índice de massa corporal: estudo pró-saúde. Rev Saúde Pública. 2004; 38(3):392-8. doi: 10.1590/S0034-89 102004000300009.

16. Pinheiro AVB, Lacerda EMA, Haimbenzecry E, Gomes MCS, Costa VM. Tabela para avaliação de consumo alimentar em medidas caseiras. Rio de Janeiro; 1994

17. Guenther PM, Kott OS, Carriquiri AL. Development of an approach for estimating usual nutrient intake distributions at the population level. J Nutr. 1997; 127(6):1106-12.

18. Carriquiri A. Assessing the prevalence of nutrient inadequacy. Public Health Nutr. 1999; 2(1):23-33. doi: $10.1017 /$ S1368980099000038.

19. Centro de Informática em Saúde da Escola Paulista de Medicina. Programa de Apoio a Nutrição, versão 2.5. Universidade Federal de São Paulo. São Paulo; 1993.

20. Tabela Brasileira de Composição de Alimentos. Versão 2. Campinas: Unicamp; 2006.

21. Fausto MA, Ansaloni JA, Silva ME, Garcia Junior J, Dehn AA, César TB. Determinação do perfil dos 
usuários e da composição química e nutricional da alimentação oferecida no restaurante universitário da Universidade Estadual Paulista, Araraquara, Brasil. Rev Nutr. 2001; 14(3):171-176. doi: 10.1590/S1415-52732001000300002.

22. Instituto Nacional de Alimentação e Nutrição. Condições nutricionais da população brasileira: adultos e idosos. Brasília: Ministério da Saúde; 1991.

23. Girotto CA, Vacchino MN, Spillmann CA, Soria JA. Prevalência de factores de riesgo cardiovascular en ingresantes universitarios. Rev Saúde Pública. 1996; 30(6):576-86. doi: 10.1590/S0034-8910 1996000600012.

24. Palma A, Abreu RA, Cunha CA. Comportamentos de risco e vulnerabilidade entre estudantes de Educação Física. Rev Bras Epidemiol. 2007; 10 (1): 117-26.

25. Guedes DP, Guedes JERP, Barbosa DS, Oliveira JÁ. Níveis de prática de atividade física habitual em adolescentes. Rev Bras Méd Esporte. 2001; 7(6): 187-99. doi: 10.1590/S1517-86922001000600 002.

26. Neumann AICP, Shirassu MM, Fisberg RM. Consumo de alimentos de risco e proteção para doenças cardiovasculares entre funcionários públicos. Rev Nutr. 2006; 19(1):19-28. doi: 10.1590/\$1415-527 32006000100002 .
27. Instituto Brasileiro de Geografia e Estatística. Pesquisa de Orçamentos Familiares (POF) 20022003: análise da disponibilidade domiciliar de alimentos e do estado nutricional no Brasil. Rio de Janeiro: IBGE; 2004.

28. Magalhães VC, Mendonça GAS. Prevalência e fatores associados a sobrepeso e obesidade em adolescentes de 15 a 19 anos das regiões Nordeste e Sudeste do Brasil, 1996 a 1997. Cad Saúde Pública. 2003; 19(1):S129-S139. doi: 10.1590/S0 102-311X2003000700014.

29. Lopes ACS, Caiaffa WT, Sichieri R, Mingoti AS, Lima-Costa MF. Consumo de nutrientes em adultos e idosos em estudo de base populacional: Projeto Bambuí. Cad Saúde Pública 2005; 21(4):1201-9. doi:10.1590/S0102-311X2005000400022.

30. Barbosa KBF, Monteiro JBR. Avaliação do consumo alimentar e sua associação com o desenvolvimento de doenças crônico degenerativas. Rev Bras Nutr Clin. 2006; 21(2):125-30.

Recebido em: 5/12/2007

Versão final reapresentada em: 17/9/2008 Aprovado em: 9/6/2009 